\title{
Prospective Study of Clinical Profile and Early Complications of Ileostomy
}

\author{
Nitesh Singh ${ }^{1}$, Pramod Kumar Bhatia ${ }^{2}$, Kirti Savyasacchi Goyal ${ }^{3}$, Sameer Pundeer ${ }^{4}$, \\ Srinivas Reddy Kallem ${ }^{5}$, Hemangi Gandhi ${ }^{6}$, Duvaraha Devi ${ }^{7}$ \\ 1, 2, 4, 5, 6,7 Department of General Surgery, MMIMSR, MMDU, Mullana, Ambala, Haryana, India. \\ ${ }^{3}$ Department of General Surgery, Government Medical College, Chandigadh, India.
}

\section{ABSTRACT}

\section{BACKGROUND}

Ileostomy is a lifesaving procedure in cases like perforation, peritonitis, typhoid fever, tuberculosis, and trauma. Ileostomy serves the purpose of diversion, decompression, and exteriorisation. Construction of ileostomy is still a common and frequently performed procedure, even though it is a major advancement in the field of surgery. This study intends to evaluate the clinical profile of ileostomy and assess early postoperative complications of ileostomy.

\section{METHODS}

A total number of 36 patients admitted in MMIMSR, Mullana (Ambala), managed and operated with ileostomy were followed up closely from the day of admission in the hospital to the day of discharge. Different parameters were studied.

\section{RESULTS}

The clinical profile, techniques, complications, indications of ileostomy were studied in detail and along with various early complications encountered with ileostomy formation. The most common complication was peristomal irritation in 33 patients (91.7\%), followed by skin excoriation in 24 patients (66.7\%), fistula in 21 patients (58.3\%), high output stoma in 17 patients (47.2\%), stomal retraction in 3 patients $(8.3 \%)$, abscess in 2 patients (5.6\%), necrosis in 1 patient $(2.8 \%)$, and stenosis in 1 patient $(2.8 \%)$.

\section{CONCLUSIONS}

Construction of ileostomy and its management was associated with high rate of complications. However, most patients tolerated the complications of ileostomy well and the overall compliance was satisfactory. Loop ileostomy was the most commonly performed stoma and was associated with high rate of complications.

\section{KEY WORDS}

Intestinal Stoma, Complications, Loop Ileostomy, End Ileostomy, Skin Excoriation, Fistula, High Output Ileostomy
Corresponding Author:

Nitesh Singh,

Room No 270, I Block,

MMU, Mullana, Ambala,

Haryana, India,

E-mail: nitesh.surgeon11@gmail.com

DOI: $10.14260 /$ jemds $/ 2021 / 149$

How to Cite This Article:

Singh N, Bhatia PK, Goyal KS, et al. Prospective study of clinical profile and early complications of ileostomy. J Evolution Med Dent Sci 2021;10(10):694699, DOI: 10.14260/jemds/2021/149

Submission 28-10-2020,

Peer Review 09-01-2021,

Acceptance 16-01-2021,

Published 08-03-2021.

Copyright (C) 2021 Nitesh Singh et al. This is an open access article distributed under Creative Commons Attribution License [Attribution 4.0 International (CC BY 4.0)] 


\section{BACKGROUND}

The word ileostomy is a derivative of 2 Greek words, eilos which means twisted bowel and stoma literally translates to mouth, which mean a hole in the twisted part of the bowel, i.e. the ileum. The further sophisticated definition of an ileostomy is a stoma constructed by bringing the end or loop of small intestine out onto the surface of skin to provides a new path to waste material to leave the body.1-2

In case of potentially dangerous anastomotic complications with an obvious risk for mortality, the role of temporary loop ileostomy is to provide defunctioning. Ileostomy is a lifesaving procedure in cases like perforation, peritonitis, typhoid fever, tuberculosis, trauma. Ileostomies solve the purpose of decompression, diversion and exteriorisation.

Complications related to ileostomy may occur early, late, intermittently or gradually \& could be acute and chronic by nature. In spite of the major improvement in the field of surgery, ileostomy construction is still a common and frequently performed surgery. It should be performed by a surgeon not only technically skilled but who also understands the potential metabolic and mechanical problems associated with the loop ileostomy. So, a judicious assessment for the need of ileostomy, careful surgical technique and skilled enterostomal nursing are essential for a satisfactory outcome. The management of a patient with ileostomy must begin before the surgery, and the key to management of surgical complications is prevention.

Early complications of ileostomy are peristomal skin irritation, which is the most common complication of ileostomy and leads to excoriation of skin. Other complications of ileostomy are obstruction, prolapse, bleeding, ischemia, retraction, stenosis, fistula formation, parastomal herniation, residual abscess, infection of wound, electrolyte imbalance, dehydration.

The basis of the current study was to evaluate the clinical profile, stomal, peristomal and other systemic complications in terms of rate, incidence and their nature during the first 6 weeks after creation of ileostomy, as most of the metabolic, mechanical and psychological complications occur during this period.

\section{METHODS}

Present study "Prospective Study of Clinical Profile and Early Complications of Ileostomy" was carried out in total of 36 patients who underwent surgical procedure and had resultant stoma (Ileostomy) in Department of General Surgery at MMIMSR, Mullana, Haryana from December 2018 to July 2020.

\section{Inclusion Criteria}

All patients admitted to Department of General Surgery undergoing exploratory laparotomy leading to ileostomy.

\section{Exclusion Criteria}

- Pregnant females.

- Patients with severe comorbidity.
- Patients who were immunocompromised like human immunodeficiency virus (HIV), hepatitis C virus (HCV).

Indication for ileostomy -

Fulminant inflammatory bowel disease.

Typhoid enteric perforation

Tuberculosis

Intestinal obstruction with gangrenous bowel

Necessity to protect of multiple or complicated anastomosis.

Multiple intestinal perforation.

Peritonitis.

Colorectal cancer

Ileostomy procedure -

Temporary ileostomies -

Permanent ileostomies -

Standard or Brooke ileostomy

Function -

Colour - Ileostomy viability was assessed by checking if the stoma is pink / red.

Degree of protrusion - It should be about $2.5 \mathrm{~cm}$. Most stomas should protrude slightly as this makes it easier to obtain a good seal when pouching.

Size - The size should be measured using the measuring guide. Skin - The peristomal skin should be healthy and intact.

Ileostomies usually function within $48-72$ hours and is initially viscous green and shiny.

\section{Early Complications}

- Retraction

- Peristomal skin irritation

- Vascular compromise

- Parastomal hernia and bowel obstruction

- Peristomal infection, abscess and fistula formation

\section{Statistical Analysis}

Data was described in terms of range; mean \pm standard deviation $( \pm \mathrm{SD})$, frequencies (number of cases) and relative frequencies (percentages) as appropriate. For comparing categorical data, chi square $(\chi 2)$ test was performed, and exact test was used when the expected frequency is less than 5 . A probability value ( $\mathrm{P}$ value) less than 0.05 was considered statistically significant. All statistical calculations were done using (Statistical Package for the Social Science) SPSS 21 version (SPSS Inc., Chicago, IL, USA) statistical program for Microsoft Windows.

\section{RESULTS}

\section{Association between Types of Ileostomy, Age Groups and Gender}

Considering the correlation between types of ileostomy, age groups and gender after constructing ileostomy we found that age group and gender were not significant with types of ileostomy as shown in following table: Table 1 


\begin{tabular}{|c|c|c|c|c|c|c|c|c|}
\hline & & & End & & oоp & Total & $\begin{array}{l}\text { Chi-Square } \\
\text { Value }\end{array}$ & $\begin{array}{c}\text { P- } \\
\text { Value }\end{array}$ \\
\hline \multirow{2}{*}{$\begin{array}{l}\text { Age } \\
\text { group }\end{array}$} & $<30$ & 5 & $38.5 \%$ & 14 & $60.9 \%$ & 19 & \multirow{2}{*}{1.673} & \multirow{2}{*}{0.196} \\
\hline & $>30$ & 8 & $61.5 \%$ & 9 & $39.1 \%$ & 17 & & \\
\hline \multirow{2}{*}{ Gender } & Female & 4 & $30.8 \%$ & 4 & $17.4 \%$ & 8 & \multirow{2}{*}{0.86} & \multirow{2}{*}{0.354} \\
\hline & Male & 9 & $69.2 \%$ & 19 & $82.6 \%$ & 28 & & \\
\hline \multicolumn{9}{|c|}{$\begin{array}{l}\text { Table 1. Association between Types of Ileostomy, } \\
\text { Age Groups, and Gender }\end{array}$} \\
\hline
\end{tabular}

\section{Intra Operative Findings}

Considering intra operative findings in patient undergoing ileostomy formation, 23 patients $(63.9 \%)$ were found to have intestinal perforation, 8 patients $(22.2 \%)$ found to have cocoon abdomen, 3 patients (8.3\%) had intestinal adhesions. Table 2.

\begin{tabular}{|ccc|}
\hline Intraop Findings & No. of Cases & Percentage \\
Cocoon abdomen & 8 & $22.2 \%$ \\
Growth & 2 & $5.6 \%$ \\
Perforation & 23 & $63.9 \%$ \\
Adhesions & 3 & $8.3 \%$ \\
Total & 36 & $100.0 \%$ \\
\hline Table 2. Distribution of Cases on the Basis of Intra Operative Findings \\
\hline
\end{tabular}
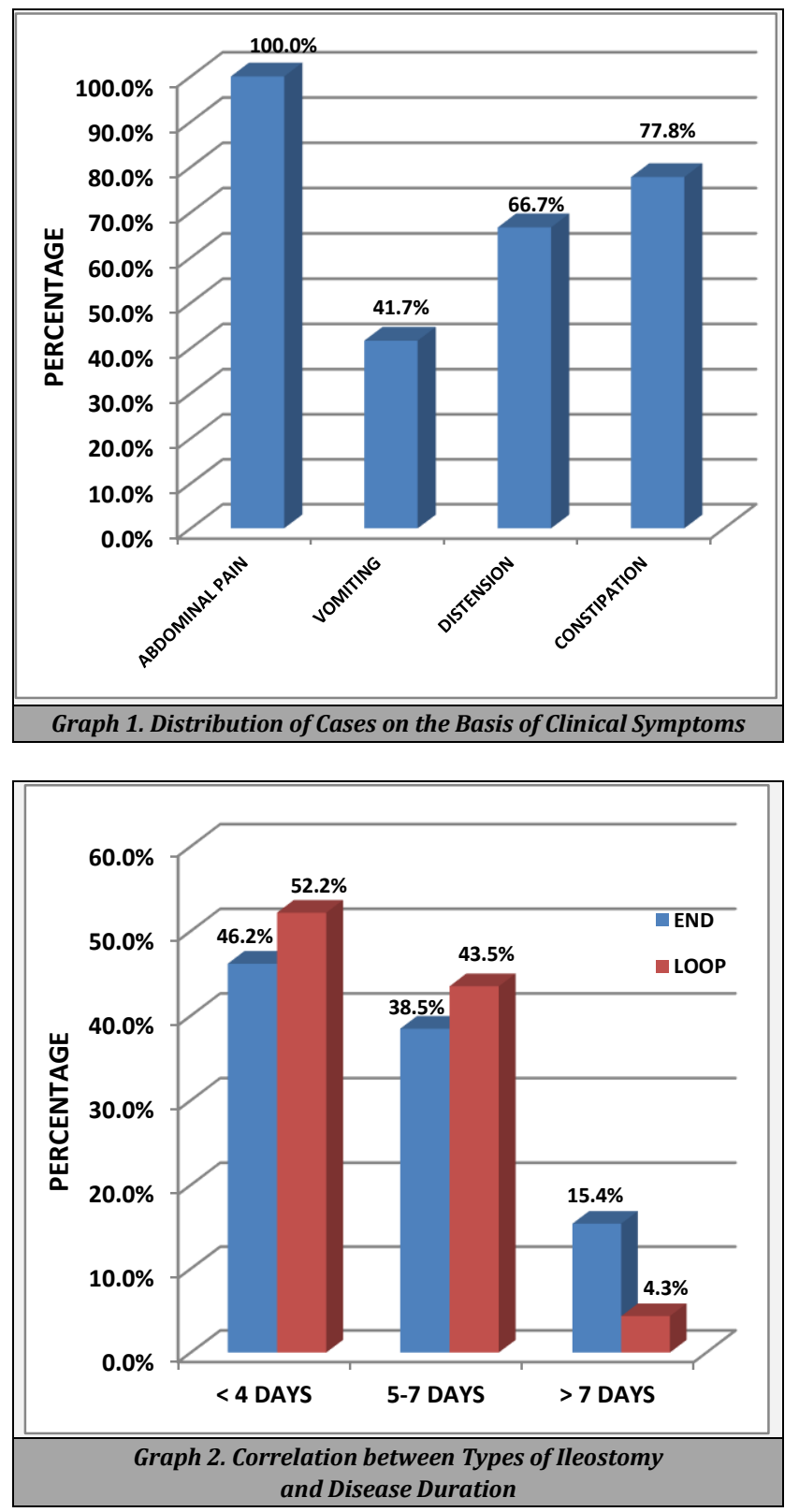

Correlation of Types of Ileostomy and Clinical Symptoms

Considering correlation of types of ileostomy and clinical symptoms. Vomiting and types of ileostomy was not found to be statistically significant $\left(x^{2}=3.306, P\right.$ value $\left.=0.069\right)$. Abdominal distension and types of ileostomy was not found to be statistically significant $\left(x^{2}=1.505, P\right.$ value $\left.=0.220\right)$. Constipation and types of ileostomy was not found to be statistically significant $\left(x^{2}=0.860, P\right.$ value $\left.=0.354\right)$. Graph 1

\section{Correlation between Types of Ileostomy and Disease Duration}

Considering correlation between types of ileostomy and disease duration was not found to be statistically significant $\left(x^{2}=1.324, \mathrm{P}\right.$ value $\left.=0.516\right)$. Graph 2

Correlation between Types of Ileostomy and Ileostomy Complications

Considering Correlation between ileostomy and complications of ileostomy, fistula and skin excoriation was most common complication with end ileostomy and was likely to be exceedingly significant $\mathrm{x}^{2}=9.663, \mathrm{P}=0.002$ and $\mathrm{x}^{2}=6.020, \mathrm{P}=$ 0.025 respectively. Table 3

\begin{tabular}{|c|c|c|c|c|c|c|c|c|}
\hline \multirow[t]{2}{*}{ Complication } & & \multicolumn{4}{|c|}{ Type of Ileostomy } & \multirow{2}{*}{\multicolumn{2}{|c|}{$\begin{array}{c}\text { Chi- } \\
\text { Total Square } \\
\text { Value }\end{array}$}} & \multirow{2}{*}{$\begin{array}{c}\text { P- } \\
\text { Value }\end{array}$} \\
\hline & & & End & & Loop & & & \\
\hline \multirow{2}{*}{ High output stoma } & - & 7 & $53.8 \%$ & 12 & $52.2 \%$ & 19 & \multirow{2}{*}{0.009} & \multirow{2}{*}{0.923} \\
\hline & + & 6 & $46.2 \%$ & 11 & $47.8 \%$ & 17 & & \\
\hline \multirow{2}{*}{ Peristomal irritation } & - & 0 & $0.0 \%$ & 3 & $13.0 \%$ & 3 & \multirow{2}{*}{1.85} & \multirow{2}{*}{0.174} \\
\hline & + & 13 & $100.0 \%$ & 20 & $87.0 \%$ & 33 & & \\
\hline \multirow{2}{*}{ Skin excoriation } & - & 1 & $7.7 \%$ & 11 & $47.8 \%$ & 12 & \multirow{2}{*}{6.020} & \multirow{2}{*}{0.025} \\
\hline & + & 12 & $92.3 \%$ & 12 & $52.2 \%$ & 24 & & \\
\hline \multirow{2}{*}{ Stomal retraction } & - & 12 & $92.3 \%$ & 21 & $91.3 \%$ & 33 & \multirow{2}{*}{0.011} & \multirow{2}{*}{0.917} \\
\hline & + & 1 & $7.7 \%$ & 2 & $8.7 \%$ & 3 & & \\
\hline \multirow{2}{*}{ Bleeding } & - & 8 & $61.5 \%$ & 16 & $69.6 \%$ & 24 & \multirow{2}{*}{0.241} & \multirow{2}{*}{0.624} \\
\hline & + & 5 & $38.5 \%$ & 7 & $30.4 \%$ & 12 & & \\
\hline \multirow{2}{*}{ Stenosis } & - & 13 & $100.0 \%$ & 22 & $95.7 \%$ & 35 & \multirow{2}{*}{0.581} & \multirow{2}{*}{0.446} \\
\hline & + & 0 & $0.0 \%$ & 1 & $4.3 \%$ & 1 & & \\
\hline \multirow{2}{*}{ Fistula } & - & 1 & $7.7 \%$ & 14 & $60.9 \%$ & 15 & \multirow{2}{*}{9.663} & \multirow{2}{*}{0.002} \\
\hline & + & 12 & $92.3 \%$ & 9 & $39.1 \%$ & 21 & & \\
\hline \multirow{2}{*}{ Necrosis } & - & 12 & $92.3 \%$ & 23 & $100.0 \%$ & 35 & \multirow{2}{*}{1.820} & \multirow{2}{*}{0.177} \\
\hline & + & 1 & $7.7 \%$ & 0 & $0.0 \%$ & 1 & & \\
\hline \multirow{2}{*}{ Abscess } & - & 12 & $92.3 \%$ & 22 & $95.7 \%$ & 34 & \multirow{2}{*}{0.177} & \multirow{2}{*}{0.674} \\
\hline & + & 1 & $7.7 \%$ & 1 & $4.3 \%$ & 2 & & \\
\hline & & & n h & & Inec & & & \\
\hline
\end{tabular}

\section{DISCUSSION}

A total of 36 patients were included in the study on basis of patient availability in given time period, as minimum number of patients required to fulfil inclusion criteria was 30. Most common age group involved in our study was $<30$ years $(52.8$ $\%$ ) followed by $>30$ years (47.2\%). Gracia et al. ${ }^{3} 2004$ also mentioned similar incidence of age.

In present study male was the predominant sex involved accounting for 28 cases (77.8 \%) and females had ileostomy in 8 cases $(22.2 \%)$. Compared to study by Pringle W et al. ${ }^{4} 2001$ where the incidence was in $62 \%$ of males and in study by Williams LA et al. 52001 incidence in $56 \%$ of males and $44 \%$ of females was reported in the study. 
Types of ileostomy in patients undergoing ileostomy formation, loop ileostomy was the most common constructed stoma, accounting for 23 patients (63.9\%) and end ileostomy was constructed in 13 patients (36.1\%).

In present study $77.8 \%$ of patient experienced constipation in loop ileostomy compared to study by Singh R et al. ${ }^{6} 2019$ where $48 \%$ patients had experienced constipation.

In present study $41 \%$ patients reported vomiting compared to study by Singh R et al. ${ }^{6} 2019$ where incidence of vomiting was experienced by $78 \%$ of patients.

In present study $100 \%$ of patients reported abdominal pain compared to study by Singh R et al. ${ }^{6} 2019$ where incidence of vomiting was experienced by $90 \%$ patients.

In present study abdominal distension was reported by $66.7 \%$ patients compared to study by Singh R et al. ${ }^{6} 2014$ where incidence of abdominal distension was experienced by $70 \%$ of patients.

Considering general physical examination i.e. pallor among patients undergoing ileostomy formation. $52.8 \%$ of patients had pallor formation compared to study by Thoker $\mathrm{M}$ et al. 72014 where examination of pallor was the most frequent finding seen in $81 \%$ of patients

Early complications occur within the first 30 days of the ileostomy formation and consist of complications like retraction, ischemia / necrosis, parastomal abscess and mucocutaneous separation. Literature reports the rate of stoma-related complications ranging from 20 to $70 \% .^{6-11}$

Higher number of complications related to ileostomy construction experienced by us and takedown by most authors. This is possibly due to operational differences in data collection and type of study carried out. Most of complications related to ileostomy in the pre-takedown period were minor and the most frequent being peristomal dermatitis and erythema.

Amongst the various early complications encountered with ileostomy formation, maximum number of complications was peristomal irritation in 33 patient ( $91.7 \%$ ), followed by skin excoriation in 24 patients (66.7 \%). Compared to complication experienced in study by Bosio et al. ${ }^{12} 2007$ there were $52 \%$ of patients who experienced skin disorder at first evaluation. Tappe et al. ${ }^{13} 2005$, the overall rate of skin problems: $25 \%$ at $0-2$ wk.; $40 \%$ at $3-6$ wk.; $20 \%$ at $7-12$ wk; $20 \%$ at $3-6$ mo; $5 \%$ at $6-12$ mo. Moreover, in present study there was significant correlation in between skin excoriation and types of ileostomy $\left(X^{2}=6.020, P=0.025\right)$.

Fistula in 21 patients (58.3\%) compared to study by Garcia-Botello et al. ${ }^{3} 2004$ where fistula experienced in $3.9 \%$ of patients. Moreover, in present study there was significant correlation in between fistula formation and types of ileostomy $\left(X^{2}=9.663, P=0.002\right)$.

High output stoma in 17 patients (47.2\%) compared to study by Cottam et al., 2007 where $34 \%$ patients experienced problematic stoma and $30 \%$ in study by Cottam et al. ${ }^{8} 2005$.

Most cases of stenosis can occur at the level of the fascia or at the level of the skin but when it occurs at the level of the skin, it mostly always causes retraction of stoma. The incidence of ileostomy retraction has been stated to range from $1-30 \%$ as it is most commonly related with colostomies and emergent operations. The incidence of stenosis is very less, ranging from $1-9 \%$. In present study ileostomy retraction was in three patients (8.3\%) compared to study by
Speirs et al. ${ }^{9} 2006$ where ileostomy retraction rate was $7.1 \%$ with bridge and $6.8 \%$ without bridge.

Stoma necrosis is most commonly associated with emergent operations, colostomies and obesity. ${ }^{21}$ Necrosis is an early postoperative complication which occurs due to inadequate stomal blood supply that can be seen in up to $13 \%$ of ostomates.

In present study necrosis was seen in 1 patient (2.8\%) compared to study by Cottam et al. ${ }^{8} 2007$ necrosis was experienced in $8.7 \%$ of patients, stenosis in 1 patient $(2.8 \%)$ compared to study done by Robertson I et al. ${ }^{10} 2005$ where stenosis was experienced in $2 \%$ of patients.

Independent factors associated with non-reversal of stomas are lower body mass index (BMI), older age, end ileostomy and preoperative radiotherapy. Prediction model indicates that there would be $64 \%$ risk of non-reversal of stoma if a patient has all risk factors. One out of three ileostomies became permanent in patients over 70 years of age. ${ }^{23}$ Similar results for end ileostomies was found in 2007 by Den Dulk et al. ${ }^{11}$ However, we could not confirm postoperative complications and recurrence to be risk factors which was found in their study.

In present study 14 (38.8\%) patients were having underweight BMI. There was significant correlation between BMI group and ileostomy $(\mathrm{x} 2=8.554, \mathrm{P}=0.014)$. However, there was no correlation in between age and ileostomy in present study $(\mathrm{P}=0.196)$

High frequency of complications associated with ileostomies could be managed mostly by minor surgical interventions. Patients with severe idiopathic constipation who are considered for an ileostomy should have a loop ileostomy through a trephine rather than a laparotomy, wherever possible.

Ileostomy closure is associated with significantly reduced postoperative nausea and vomiting, without entailing additional morbidity. In our study there were $4.16 \%$ patients experiencing vomiting compared to $22.8 \%$ in study by Worni et al. ${ }^{14} 2011$ However, vomiting and types of ileostomy was not found to be statistically significant $(\mathrm{x} 2=3.303, \mathrm{P}$ value $=$ 0.069)

In present study $82.5 \%$ of patient experienced constipation in loop ileostomy compared to study by Scarpa et al. ${ }^{15}$ was $4 \%$, patients had persistent constipation after stoma creation. However, constipation and types of ileostomy was not found to be statistically significant $(\mathrm{x} 2=0.860$, $\mathrm{P}$ value $=$ 0.354).

Complications related to ileostomy may be acute or chronic in nature and can occur early or late, intermittently or progressively. Construction of ileostomy is still a common and frequently performed procedure, despite major advancements in the field of surgery.

Complications like necrosis, skin excoriation, fistula, bleeding etc. are frequent and prevention is crucial. Therefore early, long-term and accurate follow-up is suggested, since many complications related to ileostomy may occur late after surgery and often patients are not seen routinely in the hospital at that time.

\section{Summary}

This study was conducted in all patients who came in our department with the symptoms of peritonitis, typhoid fever, 
tuberculosis, abdominal trauma, perforation distal ileal / colon obstruction or who underwent exploratory laparotomy leading to ileostomy. The findings of the study have been summarized below.

In my study 36 patients $(100 \%)$ reported abdominal pain 28 patients $(77.8 \%)$ reported constipation, 24 patients $(66.7$ $\%$ ) abdominal distension and 15 patients ( $41 \%$ ) vomiting. Age group involved in our study is $<30$ years $(52.8 \%)$ followed by $>30$ years $(47.2 \%)$.

Loop ileostomy was the most common performed accounting for 23 patients (63.9\%) and end ileostomy was constructed in 13 patients (36.1\%).

Out of the 36 patients, 14 cases (38.9\%) were underweight, 3 cases ( $8.3 \%$ ) were overweight and maximum no of cases i.e., 19 cases (52.8) underwent ileostomy were under normal BMI. Average BMI was $20.05 \pm 3.536$

On abdominal examination (palpation) in patients undergoing Ileostomy formation, 24 patients $(66.7 \%)$ had rigidity on abdominal palpation, 31 patients (86.1\%) presented with guarding, 36 patients $(100 \%)$ presented with tenderness.

Duration of symptoms in patient, 17 patients $(47.2 \%)$ presented to hospital within 4 days of onset of symptoms, while 13 patients $(36.1 \%)$ presented to hospital between 5 - 7 days of onset of symptoms. 3 patients ( $8.3 \%$ ) had symptoms for more than 7 days.

Most common complication was peristomal irritation in 33 patient (91.7\%), followed by skin excoriation in 24 patients (66.7\%), fistula in 21 patients (58.3\%), high output stoma in 17 patients ( $47.2 \%)$, stomal retraction in in 3 patients ( $8.3 \%)$, abscess in 2 patients (5.6\%), and necrosis in 1 patient (2.8\%), stenosis in 1 patient $(2.8 \%)$

In present study there was significant correlation in between skin excoriation and types of Ileostomy $\left(\mathrm{X}^{2}=6.020, \mathrm{P}\right.$ $=0.025$ ).

In present study there was significant correlation in between fistula formation and types of Ileostomy $\left(X^{2}=9.663\right.$, $\mathrm{P}=0.002$ ).

Complications like necrosis, skin excoriation, fistula, bleeding etc. are frequent and prevention is crucial. Therefore early, long-term and accurate follow-up is suggested, since many complications related to ileostomy may occur late after surgery and often patients are not seen routinely in the hospital at that time.

\section{CONCLUSIONS}

Intestinal stoma is the most commonly performed lifesaving procedure worldwide. The main reasons for creating ileostomy are to divert stool flow, protect distal anastomotic site, bowel decompression or a combination of above reasons. Indications for intestinal stoma include hollow viscus perforation, intestinal obstruction - benign or malignant, penetrating or blunt trauma abdomen, malignant disease of the large bowel (carcinoma rectum), benign disease of the large bowel (diverticular disease and inflammatory bowel disease), acute mesenteric ischemia and perianal sepsis. Benign disease is more responsible for stoma formation as compared to malignant disease and can be easily prevented.

Though ileostomy formation is a lifesaving procedure, it is associated with significant number of complications, decreased quality of life and increased economic health burden. Factors like surgical specialty, surgeon experience, stoma creation in emergency or elective hours, appropriate preoperative marking, patient's education, patient's factors like age, obesity, diabetes and other co-morbidities and finally ability to care for stoma, affect the type and frequency of complications occurring after ileostomy.

The common complications associated with stoma formation include local sepsis with excoriation, stomal necrosis, herniation, prolapse and retraction. Stomal necrosis, herniation, prolapse and retraction require stomal revision, while local excoriation demands prevention and conservative management.

Data sharing statement provided by the authors is available with the full text of this article at jemds.com.

Financial or other competing interests: None.

Disclosure forms provided by the authors are available with the full text of this article at jemds.com.

\section{REFERENCES}

[1] Doughty D. Principles of ostomy management in the oncology patient. J Support Oncol 2005;3(1):59-69.

[2] Rondelli F, Reboldi P, Rulli A, et al. Loop ileostomy versus loop colostomy for fecal diversion after colorectal or coloanal anastomosis: a meta-analysis. Int J Colorectal Dis 2009;24(5):479-88.

[3] Garcia-Botello SA, Garcia-Armengol J, Garcia-Granero E, et al. A prospective audit of the complications of loop ileostomy construction and takedown. Dig Surg 2004;21(5-6):440-6.

[4] Pringle W, Swan E. Continuing care after discharge from hospital for stoma patients. Br J Nurs 2001;10(19):127588.

[5] Williams LA, Sagar PM, Finan PJ, et al. The outcome of loop ileostomy closure: a prospective study. Colorectal Dis 2008;10(5):460-4.

[6] Singh R, Rekhi HS, Mittal S, et al. Prospective analysis of indications and complications of temporary loop ileostomy. Journal of Research and Medical Education 2014;21:27.

[7] Thoker M, Wani I, Parray FQ et al. Role of diversion ileostomy in low rectal cancer: a randomized controlled trial. Int J Surg 2014;12(9):945-51.

[8] Cottam J, Richards K, Hasted A, et al. Results of a nationwide prospective audit of stoma complications within 3 weeks of surgery. Colorectal Dis 2007;9(9):8348.

[9] Speirs M, Leung E, Hughes D, et al. Ileostomy rod--is it a bridge too far? Colorectal Dis 2006;8(6):484-7.

[10] Robertson I, Leung E, Hughes D, et al. Prospective analysis of stoma-related complications. Colorectal Dis 2005;7(3):279-85.

[11] Den Dulk M, Smit M, Peeters KCMJ, et al. A multivariate analysis of limiting factors for stoma reversal in patients with rectal cancer entered into the total mesorectal excision (TME) trial: a retrospective study. Lancet Oncol 2007;8(4):297-303. 
[12] Bosio G, Pisani F, Lucibello L, et al. A proposal for classifying peristomal skin disorders: results of a multicenter observational study. Ostomy Wound Manage 2007;53(9):38-43.

[13] Tappe AT, McKenzie F, Sheldon J, et al. Global stoma care challenges: a united approach. World Council Enterostomal Therap J 2005;25(4):15-21.
[14] Worni M, Witschi A, Gloor B, et al. Early Closure of ileostomy is associated with less postoperative nausea and vomiting. Dig Surg 2011;28(5-6):417-23.

[15] Scarpa M, Barollo M, Keighley MRB. Ileostomy for constipation: long-term postoperative outcome. Colorectal Dis 2005;7(3):224-7. 